\title{
Identificación en tiempo real de amortiguadores magneto-reológicos ${ }^{1}$
}

\author{
R.E. Jiménez-Fabián y L.A. Álvarez Icaza-Longoria \\ Instituto de Ingeniería, UNAM \\ E-mails:rjimenezf@iingen.unam.mxy alvar@pumas.iingen.unam.mx
}

\section{Resumen}

Se presenta un modelo para un amortiguador magneto-reológico utilizado en el control semiactivo de estructuras civiles. El comportamiento de este tipo de amortiguadores es descrito mediante un modelo dinámico de fricción de LuGre cuya estructura es analíticamente más simple en comparación con otros modelos presentados en la literatura. Con el modelo propuesto es posible identificar en tiempo real los parámetros del amortiguador magneto-reológico, utilizando técnicas convencionales de identificación paramétrica. Además, la fuerza que se obtiene con este modelo reproduce con muy buena aproximación las curvas que se obtienen con otros modelos de fricción desarrollados para este tipo de amortiguadores.

Descriptores: identificación de sistemas, amortiguador magneto-reológico, control semiactivo, control de estructuras, control de vibración.

\section{Abstract}

Modeling of magneto-rheological dampers to be used in semi-active control of civil engineering structures is addressed. The behavior of this kind of dampers is modeled through a first order Lugre dynamic friction model whose structure is analytically simpler than other dynamic friction models used in the literature. With this structure real-time parametric identification of the magneto-rheological damper is possible. Forces obtained with this modeling approach are in good agreement with those produced by other models.

Keywords: system identification, magneto-rheological dampers, semiactive control, structure control, vibration control.

\section{Introducción}

La protección sísmica de estructuras civiles es un tema de gran importancia en la actualidad. Las pérdidas económicas y humanas causadas por un sismo pueden llegar a ser muy grandes; por ejemplo, las trágicas consecuencias de los terremotos sucedidos en las ciudades de México, Kobe y Los Angeles ponen de manifiesto la importancia de desarrollar nuevas técnicas para atenuar la respuesta dinámica de estructuras sujetas a excitación sísmica.

Recientemente han aparecido varios trabajos enfocados al desarrollo de sistemas de control, tanto activos como pasivos, para proteger edificios y reducir los daños provocados por sismos (Ramallo et al., 1999; Ribakov y Gluk, 1999; Xu et al., 2000; Nagarajaiah et al., 2000; Zeng et al.,

1 Proyecto número $31226 \mathrm{U}$ apoyado por CONACyT 
2000). El control semiactivo combina las ventajas de ambos enfoques ofreciendo la confiabilidad de los sistemas pasivos y la adaptabilidad de los sistemas activos. En Dyke et al. (1996b) y Ramallo et al. (1999) se muestran las ventajas que ofrecen las técnicas de control semiactivo en comparación con las técnicas de control activo. Estos autores concluyen que tanto el control activo como el semiactivo logran un mejor desempeño que las técnicas de control pasivo en la proteción de edificios de varios pisos. También muestran que el desempeño de controladores activos y semiactivos es muy parecido, siendo estos últimos más baratos y confiables dada la baja potencia requerida para su funcionamiento.

En los últimos años se han propuesto varios dispositivos semiactivos para disipar la energía de vibración en sistemas estructurales. Estos dispositivos utilizan la fuerza de fricción generada en fluidos o en superficies de contacto, que en general, puede ser ajustada de alguna manera. Algunos ejemplos de estos dispositivos son los amortiguadores controlables, los amortiguadores de orificio variable, los soportes de fricción controlable, los aislamientos de fricción controlable y los dispositivos de rigidez variable (Spencer et al., 1997).

De acuerdo con Spencer et al. (1997), un dispositivo de control semiactivo es aquel que posee características que se pueden modificar en tiempo real, pero que no suministra energía al sistema controlado. Estos sistemas se caracterizan por funcionar con poca energía y porque pueden modificar el amortiguamiento estructural en la misma forma que los sistemas de control activo. El reducido consumo de energía de estos dispositivos resulta muy útil durante un sismo, cuando puede llegar a fallar el suministro de energía eléctrica.

Entre los dispositivos semiactivos recientemente desarrollados se encuentran los que utilizan fluidos controlables. En ellos la fuerza de fricción es generada en un fluido cuyas propiedades mecánicas pueden modificarse en tiempo real. La principal característica de estos dispositivos es su habilidad para cambiar reversiblemente de un estado de flujo lineal a un estado semi-sólido con una resistencia a la fluencia controlable. Los mejores candidatos para la construcción de amortiguadores controlables son los fluidos electro-reológicos y los fluidos magneto-reológicos, siendo estos últimos los más atractivos para la protección sísmica de edificios (Dyke et al., 1996b; Spencer et al., 1997).

En la práctica, es muy dificil conocer a priori el valor exacto de los parámetros de los modelos matemáticos utilizados en la ingeniería. El caso de la implantación de esquemas de control semiactivo para la protección sísmica de edificios no es la excepción. Las propiedades del a mortiguador, del fluido magneto-reológico y de la estructura controlada, pueden variar con respecto a los valores nominales, ya sea por el desgaste o por diferencias en la fabricación, en el caso de los amortiguadores, o bien, por el deterioro debido a los sismos o vientos de gran intensidad, en el caso de las estructuras. Para resolver este problema se podrían utilizar parámetros generados experimentalmente, utilizando un amortiguador del mismo tipo que en el sistema real; sin embargo, se pueden obtener mejores resultados empleando algún esquema de identificación en tiempo real que permita determinar el valor real de los parámetros.

En este artículo se presenta un nuevo enfoque para modelar el amortiguador magneto-reológico. Para describir el comportamiento del amortiguador se empleó el modelo dinámico de fricción de LuGre (Canudaset al., 1995). Las ventajas más importantes que ofrece este modelo son su capacidad de reproducir el comportamiento no lineal del a mortiguador y su simplicidad matemática en comparación con los modelos basados en la estructura de Bouc-Wen (Weng, 1976). Otra ventaja importante de este modelo es que conduce a una estructura lineal en los parámetros, dando paso al diseño de un esquema de identificación paramétrica en tiem po real.

\section{Modelado del amortiguador magneto-reológico}

En esta sección se presenta el modelado del amortiguador magneto-reológico. El primer paso en 
el diseño de estrategias de control que utilizen amortiguadores magnetoreológicos es el desarrollo de un modelo matemático capaz de reproducir el comportamiento del amortiguador. El desarrollo de este modelo representa uno de los retos más importantes, dado que los amortiguadores magnetoreológicos son altamente no lineales. Son varios los modelos que se han propuesto para el estudio de amortiguadores de fluido controlable (Dyke et al., 1996a; Spencer et al., 1997; Dyke et al., 1998; Kim y Roschke, 1999). La mayoría de estos modelos presenta deficiencias en el modelado del comportamiento no lineal del amortiguador, especialmente en la relación fuerza-velocidad; o bien, son demasiado complejos analíticamente y en ocasiones, requieren pasos de integración muy pequeños (e.g., menores a $1 \times 10^{-4} \mathrm{~s}$ ), lo que hace difícil su tratamiento numérico y reduce su utilidad práctica.

El modelado de estos amortiguadores es ampliamente analizado por Spencer et al. (1997). Para representar el comportamiento del amortiguador los autores utilizan la estructura del modelo Bouc-Wen (Weng, 1976), el cual es extremadamente versátil y puede exhibir una gran variedad de comportamientos de histéresis. Los resultados presentados en Dyke et al. (1996b) y Spencer et al. (1997) muestran que este modelo reproduce con gran precisión el comportamiento real del amortiguador bajo condiciones típicas de trabajo. El esquema del modelo presentado en Spencer et al. (1997) se muestra en la figura 1.

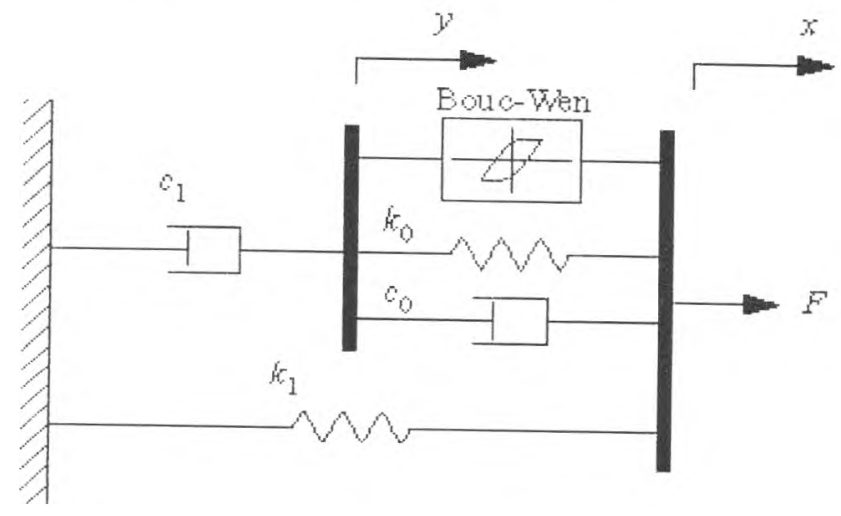

Figura 1. Representación mecánica del modelo de Spencer et al.
Las ecuaciones que gobiernan este modelo son las siguientes:

$$
\begin{gathered}
c_{1} \dot{y}=\alpha z+k_{0}(x-y)+c_{0}(\dot{x}-\dot{y}) \\
\dot{z}=-\gamma|\dot{x}-\dot{y}| z|z|^{n-1}-\beta(\dot{x}-\dot{y})|z|^{n}+A(\dot{x}-\dot{y}) \\
f=\alpha z+c_{0}(\dot{x}-\dot{y})+k_{0}(x-y)+k_{1}\left(x-x_{0}\right)
\end{gathered}
$$

En la ecuación (1) $f$ es la fuerza ejercida por el amortiguador, $x$ es el desplazamiento relativo de uno de los extremos del amortiguador ${ }^{2}$, y es un estado interno que reproduce los efectos de deslizamiento (rollover, roll-off) observados a bajas velocidades, y $z$ es el estado interno del modelo de Bouc-Wen. Las constantes $k_{0}, k_{1}, c_{0}$ y $c_{1}$ son los parámetros de rigidez y amortiguamiento viscoso de los elementos de la figura 1. Los parámetros $\gamma, \beta$ y A controlan la forma del ciclo de histéresis del modelo de Bouc-Wen. De igual forma, el parámetron está relacionado con el estado $z$ y determina su evolución. Finalmente, $\alpha$ representa el acoplamiento de $z$ con la fuerza $f$.

Para cuantificar los cambios en el esfuerzo de fluencia del fluido magneto-reológico en presencia de un campo magnético, Spencer et al. (1997) proponen las siguientes relaciones lineales entre los parámetros $\alpha, c_{0}$ y $c_{1}$ y el voltaje aplicado $u$

$$
\begin{aligned}
& \alpha=\alpha(u)=\alpha_{a}+\alpha_{b} u, \\
& c_{0}=c_{0}(u)=c_{0_{a}}+c_{0_{b}} u, \\
& c_{1}=c_{1}(u)=c_{1_{a}}+c_{1_{b}} u,
\end{aligned}
$$

donde $\alpha_{a}, \alpha_{b}, c_{o a}, c_{0 b}, c_{1 a} y c_{1 b}$ son constantes y el voltaje $u$ está dado por

$$
\dot{u}=\eta(u-v)
$$

donde $u$ representa la dinámica del fluido involucrada en alcanzar el equilibrio reológico al aplicarse un voltaje $v$ en la entrada.

El ajuste de los parámetros del modelo propuesto por Spencer et al. (1997) está basado en

2 El otro extremo está fijo en el esquema de la figura 1 
datos experimentales y muestra un desempeño muy bueno en varias pruebas de simulación. A pesar de que la respuesta de este modelo coincide con gran precisión con los resultados experimentales, su complejidad matemática limita su utilización en el diseño de esquemas de control. Además de que el modelo posee catorce parámetros, la identificación en tiempo real se dificulta dado que la estructura del modelo es no lineal en los parámetros. También es necesario mencionar que este modelo requiere de pasos de integración del orden de $2 \times 10^{-4} \mathrm{~s}$, lo que puede ser inconveniente para algunas aplicaciones prácticas.

En este trabajo se presenta un modelo dinámico basado en el modelo de fricción propuesto por Canudas et al. (1995). Este modelo tiene la siguiente forma

$$
\begin{gathered}
f=\sigma_{0} z+\sigma_{1} \dot{z}+\sigma_{2} \dot{x}, \\
\dot{z}=\dot{x}-\sigma_{0} a_{0}|\dot{x}| z,
\end{gathered}
$$

donde $f$ es la fuerza en el amortiguador magneto-reológico, $\dot{x}$ es la velocidad relativa entre los extremos, $z$ es un estado interno que modela la deformación del fluido, $\sigma_{i} ; i=0,1,2$ y $a_{0}$ son parámetros.

En el planteamiento original de Canudas et al. (1995), el parámetro $a_{0}$ es una función no lineal de la velocidad relativa ${ }^{3}$. Para mantener la simplicidad del modelo, y basándose en el modelo propuesto por Dahl (1976), el parámetro $a_{0}$ se considera constante en este trabajo.

Para incluir los efectos de la aplicación del campo magnético sobre el fluido magneto-reológico, se asume que la corriente que determina la intensidad del campo es proporcional al voltaje aplicado. Para incluir el voltaje $v$ en el modelo, las ecuaciones (4) se pueden modificar de la siguiente manera

$$
f=\sigma_{0} z v+\sigma_{1} \dot{z}+\sigma_{2} \dot{x}
$$

3 El parámetro $a_{0}$ intenta representar el efecto Stribeck (Alvarez y Olmos, 2001).

$$
\dot{z}=\dot{x}-\sigma_{0} a_{0}|\dot{x}| z\left(1+a_{1} v\right) .
$$

Finalmente, si la ecuación ( 5 b) se sustituye en (5a) se tiene

$$
f=\sigma_{0} z v-\sigma_{0} \sigma_{1} a_{0}|\dot{x}| z-\sigma_{0} \sigma_{1} a_{0} a_{1}|\dot{x}| z v+\left(\sigma_{1} \sigma_{2}\right) \dot{x} .
$$

Considerando los siguiente parámetros

$$
\begin{gathered}
\theta_{1}=\sigma_{0}, \\
\theta_{2}=\sigma_{0} \sigma_{1} a_{0}, \\
\theta_{3}=\sigma_{0} \sigma_{1} a_{0} a_{1}, \\
\theta_{4}=\sigma_{1}+\sigma_{2}
\end{gathered}
$$

y usando las ecuaciones (6) y (7) el modelo propuesto puede reparametrizarse de la siguiente manera

$$
f=U \Theta
$$

con

$$
U=[z v,-\dot{x}|z,-\dot{x}| z v, \dot{x}]^{T}
$$

y

$$
\Theta=\left[\theta_{1}, \theta_{2}, \theta_{3}, \theta_{4}\right]^{T},
$$

dando como resultado una estructura lineal en los parámetros, que es adecuada para utilizar cualquier técnica de identificación en tiempo real.

Es importante notar que sólo se pueden determinar cuatro parámetros con el vector $\Theta$, mientras que la ecuación (5) requiere cinco. Para calcular en forma única estos cuatro parámetros, se asume que el valor de $\sigma_{2}$ es conocido. El resto de los parámetros se obtiene de la siguiente forma

$$
\begin{aligned}
& \sigma_{1}=\theta_{4}-\sigma_{2}, \\
& \sigma_{0} a_{0}=\frac{\theta_{2}}{\sigma_{1}},
\end{aligned}
$$




$$
\sigma_{0} a_{0} a_{1}=\frac{\theta_{3}}{\sigma_{1}} .
$$

\section{Identificación en tiempo real del amortiguador magneto-reológico}

Una vez escrito el modelo en la forma paramétrica (8), la aplicación de cualquier algoritmo convencional de identificación es directa. En este trabajo se utilizó un algoritmo de mínimos cuadrados (Ljung, 1987; Åstrom y Wittenmark, 1995) para la identificación en tiempo real de los parámetros del modelo.

La ley de adaptación para el valor estimado $̂$ de los parámetros reales $\Theta$ es la siguiente

$$
\begin{aligned}
& \dot{P}=\alpha P-P U^{T} U P, \\
& \dot{\hat{\Theta}}=P-P U^{T} \widetilde{f},
\end{aligned}
$$

donde $\widetilde{f}=f-\hat{f}$. El regresor $U$ en la ecuación (9) requiere del conocimiento del estado interno $z$. En el modelo que aquí se propone el estado $z$ no se puede medir, por lo que se propone el siguiente observador

$$
\dot{\hat{z}}=\dot{x}-\hat{\sigma}_{0} \hat{a}_{0}|\dot{x}| \hat{z} \mid\left(1+\hat{a}_{1} v\right)
$$

donde $\hat{\sigma}_{0}, \hat{a}_{0}, \hat{a}_{1}$ son los valores estimados de los parámetros reales, calculados a través de las ecuaciones (7) y el valor estimado de $\Theta$.

El regresor $U$ es remplazado por $\hat{U}$ en la ecuación (12) donde $\hat{U}$ está definido por

$$
\hat{U}=[\hat{z} v,-|\dot{x}| \hat{z},-|\dot{x}| \hat{z} v, \dot{x}]^{T} \text {. }
$$

Substituyendo (14) en (12) se obtiene

$$
\begin{gathered}
\dot{P}=\alpha P-P \hat{U}^{T} \hat{U} P \\
\dot{\hat{\Theta}}=P-P \hat{U}^{T} \widetilde{f} .
\end{gathered}
$$

\section{Convergencia paramétrica}

Para la prueba de convergencia del esquema de identificación y del observador de $z$ considérese la siguiente función candidata de Lyapunov

$$
V=V_{1}+V_{2},
$$

donde

$$
V_{1}=\frac{1}{2} \widetilde{\Theta}^{T} P^{-1} \widetilde{\Theta}
$$

y

$$
V_{2}=\frac{1}{2} \widetilde{z}^{2}
$$

son funciones positivas definidas en las que $\widetilde{\Theta}=\Theta-\hat{\Theta}$ es el error paramétrico y $\widetilde{z}=z-\hat{z}$ es el error de observación. Para facilitar el desarrollo de la prueba de estabilidad las funciones $V_{1}$ y $V_{2}$ se tratarán por separado.

En primer lugar, si se considera el algoritmo de identificación

$$
\begin{aligned}
& \dot{P}=\alpha P-P \hat{U}^{T} \hat{U} P \\
& \dot{\hat{\Theta}}=P-P \hat{U}^{T} \widetilde{f} .
\end{aligned}
$$

la derivada con respecto al tiempo de la ecuación (17) puede escribirse como

$$
V_{1}=\frac{1}{2} \widetilde{\Theta}^{T} \hat{U}^{T} \widetilde{f}-\frac{1}{2} \widetilde{f}^{T} \hat{U} \widetilde{\Theta}+\frac{1}{2} \widetilde{\Theta}^{T} \dot{P}^{-1} \widetilde{\Theta}
$$

donde $\tilde{f}=f-\hat{f}$ es el error en la salida del esquema de identificación el cual satisface la siguiente igualdad

$$
\widetilde{f}=U \Theta-\hat{U} \Theta=\widetilde{U} \Theta+\hat{U} \Theta .
$$

Tomando en cuenta la igualdad $\dot{P}^{-1}=-P^{-1} \dot{P} P^{-1}$ y la ecuaciones (19a) y (21), la ecuación (20) queda de la siguiente manera

$$
\dot{V}_{1}=-\frac{1}{2} \widetilde{\Theta}^{T} \hat{U}^{T} \hat{U} \widetilde{\Theta}-\frac{1}{2} \alpha \widetilde{\Theta}^{T} \widetilde{\Theta}-\widetilde{\Theta}^{T} \hat{U}^{T} U_{1} \Theta \bar{z}=-\left(\widetilde{\Theta}^{T} W_{1} \widetilde{\Theta}+\widetilde{\Theta}^{T} W_{2} \widetilde{z}\right),
$$

donde

$$
W_{1}=\hat{U}^{T} \hat{U}+\alpha I,
$$




$$
W_{2}=\hat{U}^{T} \dot{U}_{1} \Theta
$$

y

$$
U_{1}=[v-|\dot{x}|-|\dot{x}| v 0]
$$

Analizando ahora la ecuación (18), la variación con respecto al tiempo del error de observación está dada por

$\dot{\widetilde{z}}=-\sigma_{0} a_{0}|\dot{x}| z-\sigma_{0} a_{0} a_{1}|\dot{x}| z v+\hat{\sigma}_{0} \hat{a}_{0}|\dot{x}| \hat{z}+\hat{\sigma}_{0} \hat{a}_{0} \hat{a}_{1}|\dot{x}| \hat{z} v$.

Sumando y restando los términos $\sigma_{0} a_{0}|\dot{x}| \hat{z}$ y $\sigma_{0} a_{0} a_{1}|\dot{x}| \hat{z} v$ a la ecuación (26) y evaluando la derivada con respecto al tiempo de la ecuación (18) se llega a

$\dot{V}_{2}=-\sigma_{0} a_{0}|\dot{x}|\left(1+a_{1} v\right) \widetilde{z}^{2}-\overline{\sigma_{0} a_{0}}|\dot{x}| \tilde{z} \widetilde{z}-\overline{\sigma_{0} a_{0} a_{1}}|\dot{x}| \tilde{z} v$.

Los últimos dos términos de la ecuación (27) están en función de los productos $\overline{\sigma_{0} a_{0}}$ y $\overline{\sigma_{0} a_{0} a_{1}}$ cuyo signo no puede determinarse fácilmente. Para evitar este problema es conveniente expresar estos productos en términos de valores conocidos. Por ejemplo, utilizando la ecuación (11b), el término $\overline{\sigma_{0} a_{0}}$ puede expresarse de la siguiente manera

$$
\begin{gathered}
-\overline{\sigma_{0} a_{0}}=\hat{\sigma}_{0} \hat{a}_{0}-\sigma_{0} a_{0} \\
=\frac{\hat{\theta}_{2}}{\hat{\sigma}_{1}}-\frac{\theta_{2}}{\sigma_{1}} \\
=\frac{\sigma \theta_{2}-\sigma_{1} \hat{\theta}_{2}}{\sigma_{1} \hat{\sigma}_{1}} .
\end{gathered}
$$

Sustituyendo $\hat{\sigma}_{1}=\sigma_{1}-\widetilde{\sigma}_{1}$ en (30) y reagrupando se tiene

$$
-\overline{\sigma_{0} a_{0}}=\frac{\sigma_{1}\left(\hat{\theta}_{2}-\theta_{2}\right)+\theta_{2} \widetilde{\sigma}_{1}}{\sigma_{1} \hat{\sigma}_{1}}
$$

Además, dado que $\sigma_{2}$ se supone conocido, $\widetilde{\theta}_{4}=\theta_{4}-\hat{\theta}_{4}=\sigma_{1}+\sigma_{2}-\hat{\sigma}_{1}-\sigma_{2}=\widetilde{\sigma}_{1}$. Con este último resultado, la ecuación (31) finalmente se reduce a

$$
-\overline{\sigma_{0} a_{0}}=\frac{\sigma_{1}\left(\hat{\theta}_{2}-\theta_{2}\right)+\theta_{2} \widetilde{\theta_{4}}}{\sigma_{1} \hat{\sigma}_{1}}=-\frac{1}{\hat{\sigma}_{1}} \widetilde{\theta}_{2}+\frac{\theta_{2}}{\sigma_{1} \hat{\sigma}_{1}} \widetilde{\theta}_{4} .
$$

Siguiendo el mismo procedimiento se obtiene una siguiente expresión equivalente para el producto $\overline{\sigma_{0} a_{0} a_{1}}$

$$
-\overline{\sigma_{0} a_{0} a_{1}}=-\frac{1}{\hat{\sigma}_{1}} \widetilde{\theta}_{3}+\frac{\theta_{3}}{\sigma_{1} \hat{\sigma}_{1}} \widetilde{\theta}_{4} .
$$

Con el desarrollo algebraico anterior se logra expresar la ecuación (27) de la siguiente manera

$\dot{V}_{2}=-\sigma_{0} a_{0}|\dot{x}|\left(1+a_{1} v\right) \widetilde{z}^{2}+|\dot{x}| \hat{z}\left[-\frac{1}{\hat{\sigma}_{1}} \widetilde{\theta}_{2}-\frac{v}{\hat{\sigma}_{1}} \widetilde{\theta}_{3}+\frac{\theta_{2}+\theta_{3} v}{\sigma_{1} \hat{\sigma}_{1}} \widetilde{\theta}_{4}\right] \widetilde{z}$

$$
=W_{3} \widetilde{\Theta}-W_{4} \widetilde{z}^{2},
$$

donde

$$
W_{3}=\left[0, \frac{1}{\hat{\sigma}_{1}}, \frac{1}{\hat{\sigma}_{1}} v,-\frac{\theta_{2}+\theta_{3} v}{\sigma_{1} \hat{\sigma}_{1}}\right] \dot{x} \mid \hat{z}
$$

y

$$
W_{4}=-\sigma_{0} a_{0}|\dot{x}|\left(1+a_{1} v\right) .
$$

Utilizando las ecuaciones (22) y (38), la derivada con respecto al tiem po de la ecuación (16) toma la siguiente forma

$$
V=-\left(\widetilde{\Theta}^{\mathrm{T}} W_{1} \widetilde{\Theta}+\widetilde{\Theta}^{\mathrm{T}} W_{2} \widetilde{z}+W_{3} \widetilde{\Theta} \widetilde{z}+W_{4} \widetilde{z}^{2}\right)
$$

o bien,

$$
\dot{V} \leq-\left(\widetilde{\Theta}^{T} W_{1} \widetilde{\Theta}+\widetilde{\Theta}^{T} W_{2} \widetilde{z}+W_{3} \widetilde{\Theta} \widetilde{z}+W_{4} \widetilde{z}^{2}\right),
$$

donde $\bar{W}_{1}=\hat{U}^{T} \hat{U}$ si se desprecia el término $\alpha I$ en (23).

La expresión (38) constituye una forma cuadrática dada por

$$
\dot{V} \leq-[\widetilde{\Theta} \widetilde{z}]\left[\begin{array}{ll}
\bar{W}_{1} & W_{2} \\
W_{3} & W_{4}
\end{array}\right]\left[\begin{array}{l}
\widetilde{\Theta} \\
\widetilde{z}
\end{array}\right]
$$

en la que los determinantes principales de 


$$
\left[\begin{array}{ll}
\bar{W}_{1} & W_{2} \\
W_{3} & W_{4}
\end{array}\right]
$$

son todos iguales a cero, excepto el de menor orden que es igual a $v^{2} \hat{z}^{2}>0$, con lo que se puede concluir directamente que (40) es negativa semidefinida, demostrándose así que los puntos $\widetilde{f}=0$ y $\widetilde{z}=0$ son equilibrios estables.

Normalmente la fuerza inducida por ondas sísmicas es suficientemente rica en frecuencias, garantizando excitación persistente, con lo que en la mayoría de los casos prácticos $\widetilde{\theta}=0$.

\section{Resultados de simulación}

Para evaluar el desempeño del modelo propuesto, se utilizó el modelo presentado en Spencer et al. (1997) como referencia para generar la señal de fuerza considerada como salida en el esquema de identificación. Los valores de los parámetros de las ecuaciones (1a-c) fueron tomados de Spencer et al. (1997) y se muestran en la tabla 1.

Tabla 1. Parámetros para el modelo de las ecuaciones $(1 \mathrm{a}-\mathrm{c})$

\begin{tabular}{cc}
\hline Parámetro & Valor \\
\hline$c_{0_{n}}$ & $20.2 \mathrm{~N} \mathrm{~s} / \mathrm{cm}$ \\
$c_{0_{r}}$ & $2.68 \mathrm{~N} \mathrm{~s} / \mathrm{V} \mathrm{cm}$ \\
$k_{0}$ & $15.0 \mathrm{~N} \mathrm{~cm}$ \\
$c_{1,}$ & $350 \mathrm{~N} \mathrm{~s} / \mathrm{cm}$ \\
$c_{1}$ & $70.7 \mathrm{~N} \mathrm{~s} / \mathrm{V} \mathrm{cm}$ \\
$k_{1}$ & $5.37 \mathrm{~N} / \mathrm{cm}$ \\
$\alpha_{a}$ & 44.9 \\
$\alpha$ & $6381 / \mathrm{V}$ \\
$\gamma$ & $39.31 / \mathrm{cm}^{2}$ \\
$\beta$ & $39.31 / \mathrm{cm}^{2}$ \\
$\mathrm{~A}$ & 47.2 \\
$n$ & 2 \\
$\eta$ & $2511 / \mathrm{s}$ \\
\hline \hline
\end{tabular}

El primer resultado de simulación corresponde a una señal de entrada en la que el desplazamiento $x[\mathrm{~cm}]$ y el voltaje de entrada $v[V]$ satisfacen

$$
x=1.15 \sin (8.6 \pi \cdot t)
$$

$$
\mathrm{y} \quad v=1.25+1.25 \sin (10.2 \pi \mathrm{t})
$$

Usando estas señales de entrada se utilizan las ecuaciones (1a-c) para generar una señal de referencia $f$ para el esquema de identificaciónobservación. Las curvas obtenidas se muestran en la figura 2 . Se puede observar una muy buena aproximación de la fuerza generada por el modelo propuesto.

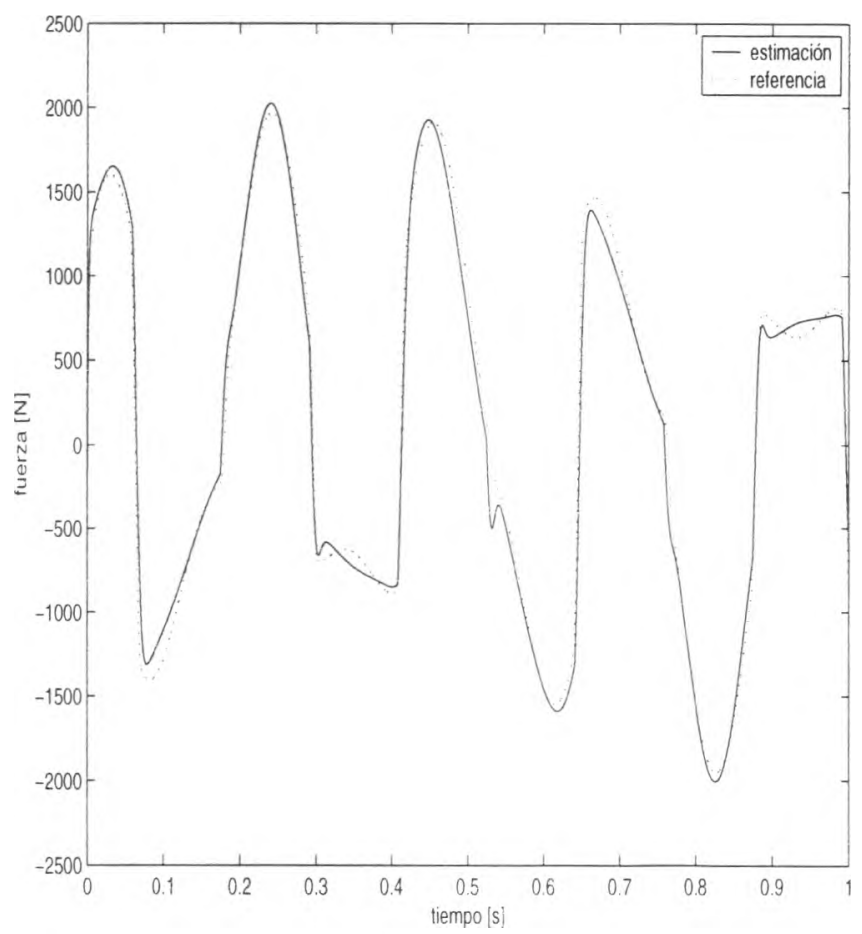

Figura 2. Respuesta del modelo del amortiguador a una señal de excitación variante en el tiempo

El vector de parámetros estimados que se obtiene después de $36 \mathrm{~s}$ de simulación es

$$
\hat{\theta}=10^{4} \times[1.059,0.181,-0.026,0.008]^{T} .
$$

La evolución de los parámetros se muestra en la figura 3. En esta gráfica se puede observar que el tiempo de respuesta del sistema es aproximadamente de $1.5 \mathrm{~s}$.

En el segundo experimento de simulación, los parámetros de la ecuación (41) se mantuvieron constantes para probar el desempeño del modelo bajo diferentes señales de excitación. La señal de entrada, tanto para el modelo dado por las 
ecuaciones ( $1 \mathrm{a}-\mathrm{c}$ ) como para el modelo dado por la ecuación (5), fue un desplazamiento senoidal $x[\mathrm{~cm}]$ tal que

$$
x=1.5 \sin (5 \pi \cdot t)
$$

con voltajes de entrada de $0.75,1.5$ y 2.25 [V]. Las figuras 4-6 muestran los resultados obtenidos para cada voltaje. La figura 4 muestra la evolución de la fuerza en el tiempo, la figura 5 presenta la relación fuerza-desplazamiento y, finalmente, la figura 6 muestra la curva fuerza vs. velocidad.

En resumen, los resultados mostrados en las figuras 2-6 muestran un buen desempeño del modelo propuesto en comparación con la fuerza de referencia generada con el modelo Bouc-Wen. La convergencia de los parámetros es también muy buena, como se muestra è la figura 3 .
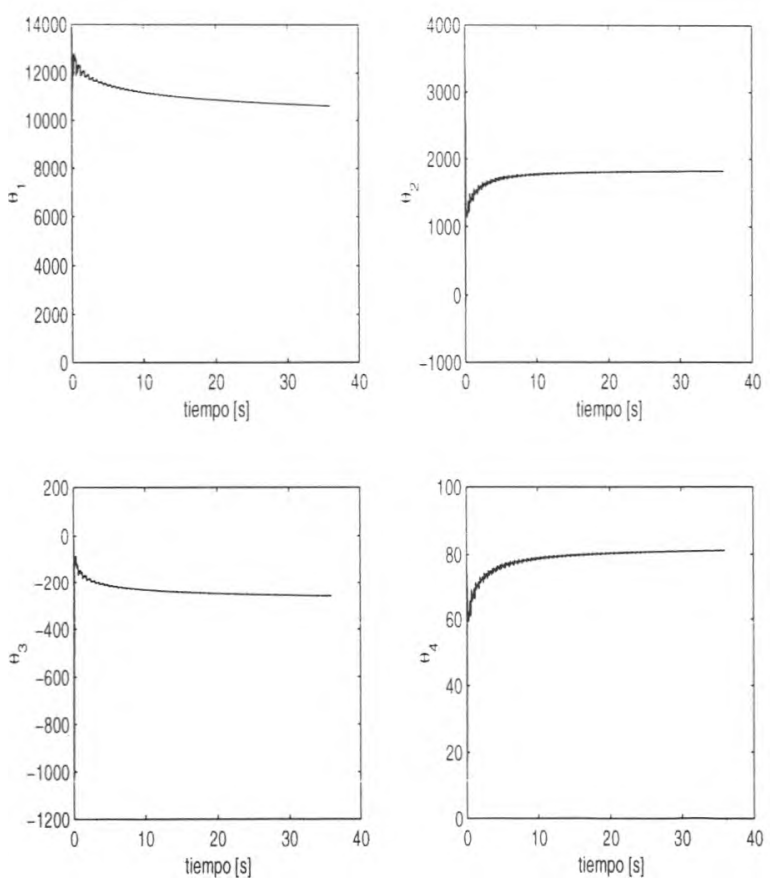

Figura 3. Evolución de los parámetros estimados vs. tiempo

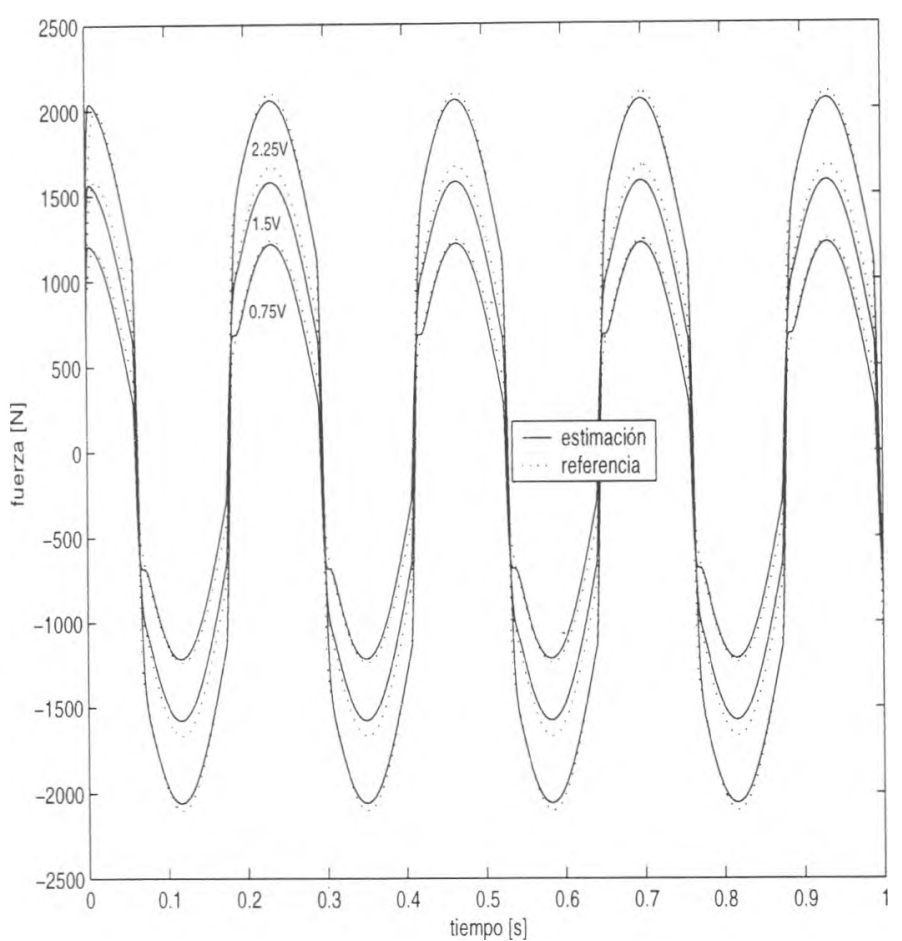

Figura 4. Fuerzas vs. tiempo para diversos voltajes de entrada constantes

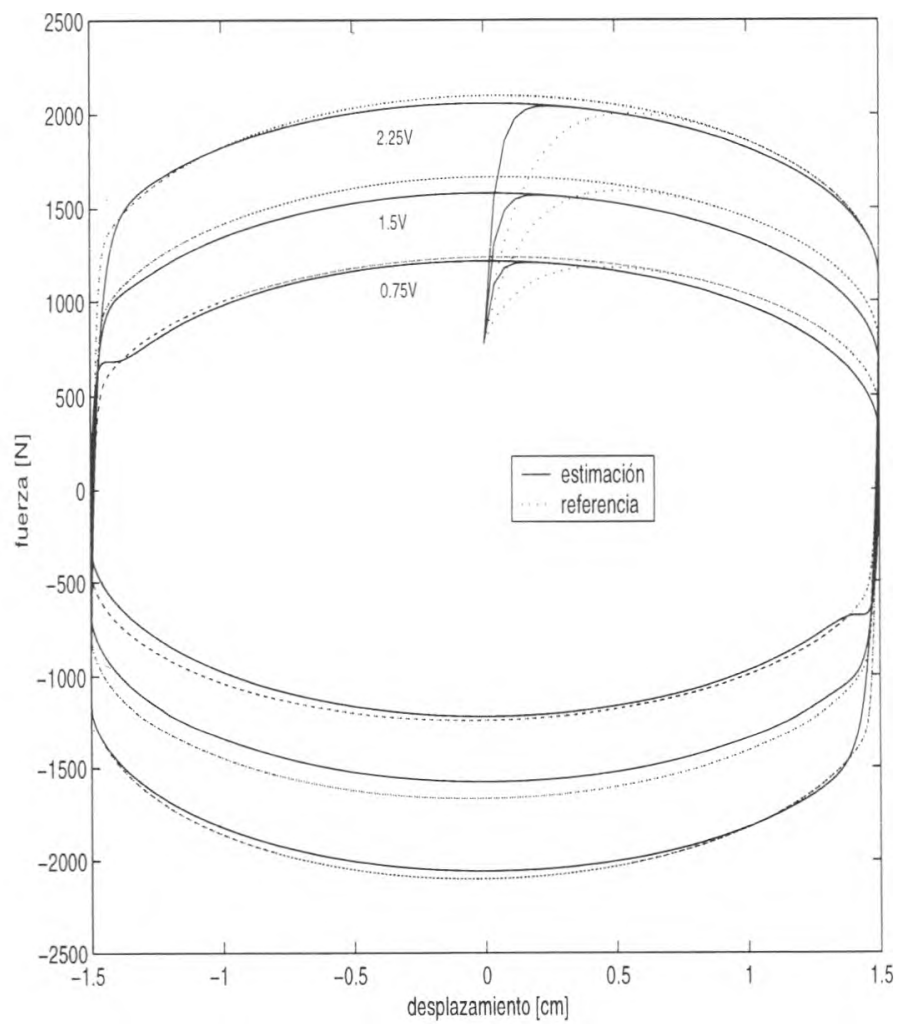

Figura 5. Fuerza vs. desplazamiento para diversos voltajes de entrada constantes 


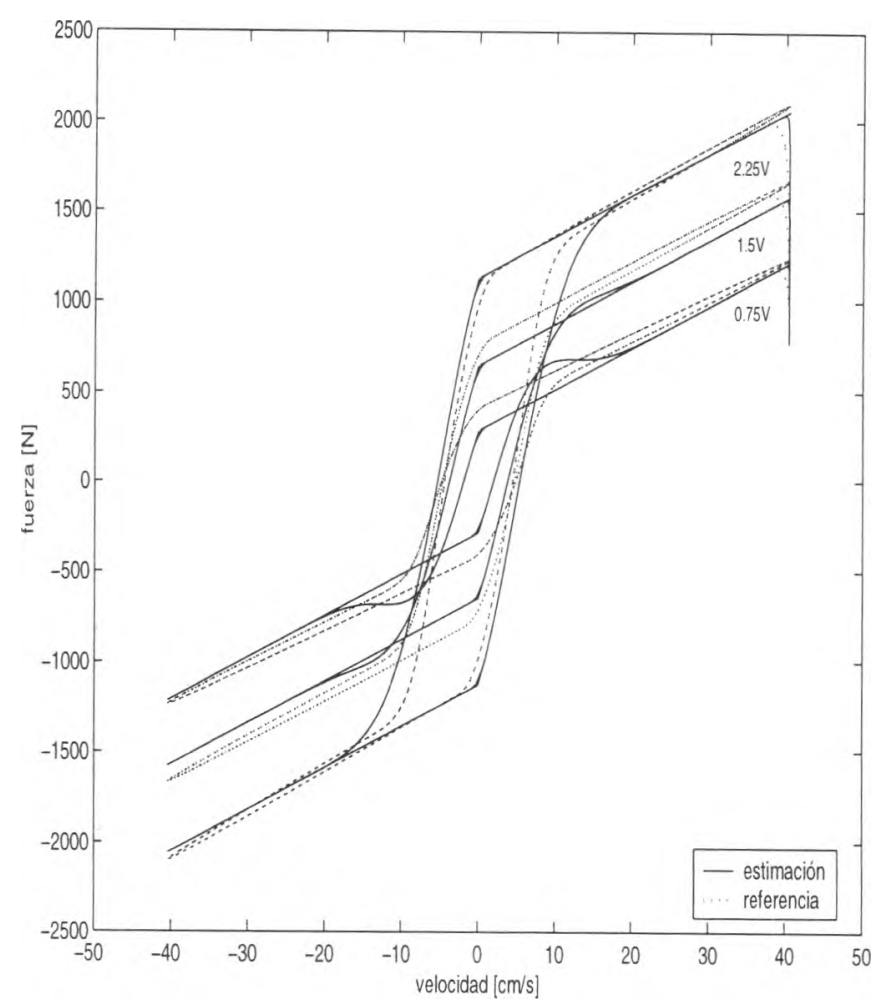

Figura 6. Fuerza vs. velocidad para diversos voltajes de entrada constantes

\section{Conclusiones}

En este trabajo se presenta un nuevo enfoque para modelar amortiguadores magneto-reológicos. El modelo presentado se basa en un modelo dinámico de fricción de primer orden de Lugre. La estructura propuesta puede transformarse en un modelo lineal en los parámetros que, en conjunto con un observador no lineal para el estado interno del modelo, permite estimar en tiempo real los parámetros del amortiguador. Una de las principales ventajas del modelo propuesto, es que posee una estructura analíticamente más simple en comparación con la que se presenta en Spencer et al. (1997), pero es capaz de reproducir adecuadamente la fuerza generada en el amortiguador sujeto a diferentes entradas. Otra característica importante del modelo propuesto es que, bajo ciertas consideraciones, permite identificar todos sus parámetros en tiemo real. La obtención de datos experimentales queda como trabajo futuro para la validación de los resultados aquí presentados.

\section{Referencias}

Alvarez L. y Olmos L. (2001). Observador y adaptación paramétrica de un modelo dinámico de fricción llanta/pavimento. Memorias del Segundo Taller Nacional de Observación y Estimación en Sistemas no Lineales. C. Verde y J. Moreno eds. Series del Instituto de Ingeniería. 189-196.

Canudas C., Olsson H., Åstrom, K.J. y Lischinsky P. (1995). A New Model for Control of Systems with Friction. IEEE Transactions on Automatic Control, Vol. 40:3, 419-425.

Dahl P. (1976). Solid Friction Damping of Mechanical Vibrations. AIAA Journal, Vol. 14:12, 1675-1682.

Dyke S., Spencer B., Sain M. y Carlson J. (1996a). Modelling and Control of Magnetorheological Dampers for Seismic Response Reduction. Smart Materials and Structures, Vol. 5, 565-575.

Dyke S., Spencer B., Sain M. y Carlson J. (1996b). Seismic Response Reduction Using Magnetorheological Dampers. Proceedings of the 1996 IFAC World Congress, San Francisco, California, Vol. L, 145-150.

Dyke S., Spencer B., Sain M. y Carlson J. (1998). An Experimental Study of MR Dampers for Seismic Protection. Smart Materials and Structures, Special Issue on Large Civil Structures, Vol. 7, 693-703.

Kim B. y Roschke P. (1999). Linearization of Magnetorheological Behavior Using a Neural Network. Proceedings of the American Control Conference, San Diego, California\}, 4501-4505. Ljung L. (1987). System Identification: Theory for the user. Prentice-Hall, USA.

Nagarajaiah S., Sahasrabudhe S. y Iyer R. (2000). Seismic Response of Sliding Isolated Bridges with MR Dampers. Proceedings of the American Control Conference, Chicago, Illinois, 4437-4441.

Åstrom K. y Wittenmark B. (1995). Adaptive Control, 2a. ed. Addison-Wesley, USA.

Ramallo J., Jonhson E., Spencer B. y Sain M. (1999). Semiactive Building Base Isolation. Proceedings of the American Control Conference, San Diego, California, 515-519. 
Ribakov Y. y Gluk J. (1999). Active Control of MDOF Structures with Supplemental Electrorheological Fuid Dampers. Earthquake Engineering and Structural Dynamics, Vol. 28, 143-156.

Spencer B., Dyke S., Sain M. y Carlson J. (1997). Phenomenological Model of a Magnetorheological Damper. ASCE Journal of Engineering Mechanics, Vol. 123:3, 230-238.

Weng Y. (1976). Method for Random Vibration of Hysteric Systems. ASCE Journal of Engineering Mechanics, Vol. 102 (EM2), 249-263.
Xu Y., Qu W. Y Ko J. (2000). Seismic Response Control of Frame Structures Using Magnetorheological / Electrorheological Dampers. Earthquake Engineering and Structural Dynamics, Vol. 29, 557-575.

Zeng H., Kuehn G., Song G., Sun H. y Stalford J. (2000). A Semi-active Controller for the Vibration Absorber on the I-35 Walnut Creek Bridge. Proceedings of the American Control Conference, Chicago, Illinois, 3565-3569.

\section{Semblanza de los autores}

René E. Jiménez-Fabián. Es estudiante de doctorado del Programa de Maestría y Doctorado en Ingeniería de la UNAM. Realizó sus estudios de ingeniería mecánica en la Facultad de Ingeniería de la UNAM y obtuvo su maestría en Ingeniería Eléctrica (control) en el Programa de Maestría y Doctorado en Ingeniería de la UNAM. Su línea de investigación doctoral se refiere al control semiactivo de estructuras civiles.

Luis A. Álvarez Icaza-Longoria. Es investigador titular de la Coordinación de Automatización del Instituto de Ingeniería de la UNAM. Imparte cátedra en la División de Ingeniería Eléctrica de la Facultad de Ingeniería y en los posgrados de Ingeniería y Ciencias de la Computación, todos de la UNAM. Obtuvo su licenciatura en ingeniería electrómecaníca y su maestría en ingeniería en la Facultad de Ingeniería de la UNAM. Cursó sus estudios de doctorado en ingeniería mecánica en la Universidad de California en Berkeley. Sus líneas de investigación se refieren principalmente al control de sistemas no-lineales con aplicaciones al control de tráfico vehicular, control avanzado de vehículos y control de estructuras civiles. 